\title{
ÉTUDE EXPÉRIMENTALE CHEZ LE RAT D'OVAIRES RENDUS DYSGÉNÉSIQUES DURANT LEUR DÉVELOPPEMENT EMBRYONNAIRE
}

\author{
Elisabeth VANHEMS et Jacqueline BOUSQUET \\ avec la collaboration technique de Mllo MarTiN \\ Laboratoire d'Histologie et Embryologie, \\ (Professeur G. MAYER), \\ UER 3, Université de Bordeaux II, \\ 146, rue Léo-Saignat, \\ 33076 Bordeaux Cédex (France)
}

\section{RÉSUMÉ}

Des ovaires dysgénésiques ont été obtenus en détruisant les cellules germinales pendant 13 vie embryonnaire par injection de Misulban à la Ratte gestante. En l'absence d'ovocytes l'organisation folliculaire ne se $\mathrm{f}$ it pas et l'ovaire présente une architecture cordonnale qu'il conserve après la puberté. S'il subsiste des cellules germinales, des follicules évoluent en présentant le plus souvent une hypertrophie théc lle : le tissu théco-interstitiel augmente avec l'âge des animaux alors que les structures cordonnales diminuent ou disparaissent. Les frottis vaginaux, la structure des effecteurs témoignent d'une imprégnation hormonale anormale. Ces ovaires dysgénésiques greffés dans le rein de rattes castrées répondent aux stimulations gonadotropes en fonction des constituants qu'ils possèdent au moment de la greffe. Les cordons ne sont pas modifiés en $I_{5}$ jours de greffe. Li fouction pituitaire de ces animaux, testée par la méthode des greffes, montre une hypersécrétion gonadotrope.

\section{INTRODUCTION}

L'expérimentation a montré qu'en général, les fotus de Souris et de Rat irradiés avec une dose de rayons $X$ suffisante, devenaient stériles après la naissance (cf. RAYNAUd et FriLiEY, I946-47 ; RUGH, I9j2 ; MinTz, I958 ; RUGH et JACKSON, I958, chez la Souris), (MANDL, I959-63-64; BEAUMONT, I96I, chez le Rat). 
Parmi les substances antitumorales, certaines appelées radiomimétiques exercent sur les cellules germinales une action semblable à celle produite par les radiations. Ce sont en particulier :

- Le Cyclophosphamide, dont l'action nocive sur les cellules sexuelles a été mise en évidence par GERLINGER (Ig66) sur le Lapin.

- Le Melphalan, dont Pageot-Simpson et WolfF (r969) ont révélé 1'action destructrice sur les gonocytes embryonnaires de Poulet.

- Le Misulban (I-4 diméthane sulfonyloxybutane), surtout utilisé en clinique humaine en vue d'un traitement chimiothérapeutique des leucémies myéloïdes chroniques et dont MAUSS et MEYER (I964), HELLER et JONES (I963-64), HeMwORTh et JACKSON (I962-63-65-69), FORSBERG (Ig66), ont montré qu'il exerce une action néfaste sur la lignée germinale chez le Rat, PinTo-Machado (I966) chez la Souris. DiAmond, ANDERson et Creadie (Ig6o) ont aussi décrit une hypoplasie ovarienne chez un enfant né d'une mère leucémique traitée par le Misulban.

Afin d'examiner chez le Rat, l'évolution de la structure et l'activité endocrine d'ovaires rendus dysgénésiques durant leur développement embryonnaire, nous avons provoqué des dysgénésies en détruisant les cellules germinales chez le fœtus par action du Misulban injecté à la mère. Nous avons étudié le retentissement du traitement - sur la structure de l'ovaire des animaux issus de mères traitées à différentes périodes de la gestation pour tenter de déterminer la période de sensibilité maximale des cellules germinales - sur l'évolution et la fonction de l'ovaire en l'absence de cellules germinales - sur sa réactivité aux hormones gonadotropes enfin sur la fonction pituitaire de l'animal dysgénésique.

\section{MATÉRIEL E'T TECHNIQUES}

Les animaux utilisés sont des rats Wistar, entretenus dans un local à température constante $\left(25^{\circ}\right)$, éclairé artificiellement et régulièrement ( ${ }_{4}$ heures de lumière pour Io heures d'obscurité). Les animaux sont nourris avec une alimentation standard et reçoivent de l'eau à volonté.

Trois cents rattes pesant de 180 à 300 grammes ont été isolées le premier jour de la gravidité, mis en évidence par la découverte du bouchon vaginal. Ces rattes ont reçu une injection intrapéritonéale de Misulban, la substance pure fut mise en suspension dans l'huile d'arachide à raison de $2 \mathrm{mg} / \mathrm{ml}$, la quantité administrée étant de $10 \mathrm{mg} / \mathrm{kg}$. L’injection a été réalisée le matin entre ro heures et $\mathrm{I}$ I heures et à diverses périodes de la gestation : du $9^{\mathrm{e}}$ au $\mathbf{~} 7^{\mathrm{e}}$ jour afin de déterminer la période de grande sensibilité de l'ébauche des gonades.

Nous avons laissé évoluer la gestation a fin d'obtenir des nouveau-nés et de les élever. Nous avons sacrifié les femelles issues des mères traitées à différentes périodes :

- dans les jours qui suivent la naissance a fin d'observer l'évolution des gonocytes restants,

- en période impubère, pour suivre l'organisation ovarienne,

- après la puberté, pour étudier si l'ovaire peut être le siège d'ovulation et d'édification de corps jaunes, et pour explorer le développement du tissu théco-interstitiel.

Chez la femelle adulte issue de mères injectées le $13^{\mathrm{e}}$ ou le $\mathbf{5} 5^{\mathrm{e}}$ jour de la gestation, l'étude du cycle vaginal, celle de l'aptitude à la reproduction et de la structure des effecteurs nous ont permis d'aborder l'examen de la fonction des ovaires dysgénésiques obtenus.

Pour compléter ces résultats, nous avons recherché d'une part les potentialités réactionnelles d'ovaires dysgénésiques de la période périnatale à l'égard d'hyperstimulations pituitaires, en les greffant sous la capsule rénale gauche de femelles adultes normales, castrées le jour de la greffe. D'autre part, afin de savoir si le dysfonctionnement est essentiellement ovarien, nous avons 
testé la fonction pituitaire de ces animaux en greffant un ovaire normal du jour de la naissance sous la capsule rénale gauche de femelles dysgénésiques âgées de 70 jours. Les greffons sont laissés en place 15 jours ou I mois. Au terme de l'expérience, le greffon et le tractus génital sont soumis à un examen histologique.

\title{
RÉSULTATS
}

\author{
A. - Évolution post-natale des embryons femelles \\ dont la mère a été traitée par une injection de Misulban
}

\section{I. Étude des ovaires dysgénésiques de la période périnatale.}

Les ovaires des animaux issus de mères traitées sont plus petits que les témoins du même âge, leur dissection est toujours difficile.

A l'examen microscopique et quelle que soit la date de l'injection du $9^{\text {e }}$ au I6 $6^{\mathrm{e}}$ jour inclus de la gestation, on observe toujours une diminution du nombre des cellules germinales. Les altérations sont plus importantes lorsque l'injection a été réalisée à partir du $\mathrm{I} 2^{\mathrm{e}}$ jour de la gestation et il existe un maximum de destruction très net si le traitement est effectué $1 \mathrm{l} I 5^{\mathrm{e}}$ jour. La sensibilité des gonocytes au Misulban diminue après le $16^{\mathrm{e}}$ jour. Les ovaires de rattes issues de mères injectées

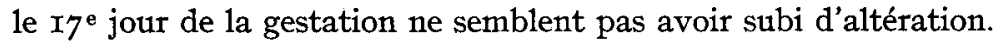

Deux ou trois jours après la naissance, les cellules germinales qui ont échappé au traitement s'accroissent et des follicules primordiaux s'organisent comme dans un ovaire normal. Leur nombre est fonction de l'importance de la destruction pendant la vie embryonnaire. En l'absence de cellules germinales, les cordons cellulaires ne se fragmentent pas, l'individualisation folliculaire ne se fait pas et l'aspect trabéculaire de l'ovaire persiste.

\section{2. Étude des ovaires dysgénésiques de la période impubère.}

Comme dans un ovaire normal, les follicules primordiaux rescapés évoluent en follicules primaires par prolifération des cellules folliculeuses autour de l'ovocyte.

Si l'altération de la gonade est grave, en particulièr si le traitement a été effectué

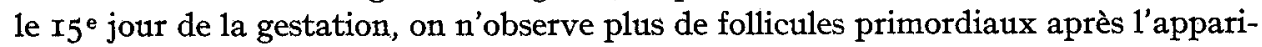
tion des follicules primaires. Il semble qu'en raison de leur faible nombre, leur évolution en follicules primaires ait été immédiate, il n'y a donc pas de réserve folliculaire comme dans un ovaire normal.

En l'absence totale de follicules, le parenchyme ovarien présente la même structure trabéculaire à direction radiaire en doigts de gant, il conserve donc la structure générale qu'il avait pendant la vie postnatale. Ces structures cordonnales voisinent avec des follicules dans le cas où la destruction n'a pas été totale.

Les follicules primaires peuvent s'accroître normalement, la formation de l'antrum aboutit au stade de follicule cavitaire, et même de follicule mûr, la thèque se différencie autour du follicule et apparaît hypertrophiée. Elle est constituée de grosses cellules à noyau arrondi. A un stade plus avancé, cette hypertrophie de la thèque aboutit à la formation de "nodules thécaux " donnant à l'ovaire un aspect très dense. 


\section{3. Étude des ovaires dysgénésiques après la puberté.}

Des ovaires dysgénésiques observés à 50 jours, présentent parfois des corps jaunes caractéristiques, issus de la transformation d'un follicule. Mais souvent, quand l'injection a été réalisée le $15^{\mathrm{e}}$ jour de la gestation, les corps jaunes que l'on peut observer sont très petits par rapport à ceux d'ovaires témoins.

En l'absence totale de follicules et de corps jaunes, l'ovaire conserve une architecture " cordonnale " dans laquelle deux types cellulaires sont représentés : les premières identiques aux cellules constituant les cordons déjà décrits, les autres organisées aussi en travées ou nappes plus ou moins diffuses sont volumineuses. On peut aussi observer des formations cordonnales bien délimitées au sein desquelles deux catégories de cellules sont représentées.

A partir de 3 mois, tout le tissu thécal est constitué de cellules volumineuses, les follicules sont de plus en plus rares, quelques structures en cordons persistent. L'ovaire devient de plus en plus dense, épais, uniquement constitué de grandes cellules polyédriques ou sphériques d'aspect floride, cytologiquement actives, avec un noyau volumineux, sphérique, possédant un nucléole bien marqué. Deux types cellulaires se différencient : l'un à cytoplasme bien colorable, 1'autre à cytoplasme clair, spumeux. Ces cellules peuvent être isolées ou groupées en amas très denses. Les structures en cordons sont en régression dans la majorité des cas et situées le plus souvent à la périphérie de l'ovaire.

Ces images s'accentuent à des stades plus avancés. Nous les avons observées jusqu'à I4 mois.

\section{B. - Fonction de l'ovaire dysgénésique}

L'aptitude à la reproduction des femelles dysgénésiques, les frottis vaginaux, l'étude histologique des effecteurs, nous ont renseignés sur le fonctionnement des ovaires. Ces tests ont été effectués sur des rattes issues de mères ayant reçu une injection unique de Misulban le $13^{\mathrm{e}}$ ou le $15^{\mathrm{e}}$ jour de la gestation.

Ces dates ont été choisies en fonction des premiers résultats, 1 e $15^{\circ}$ jour nous permettant d'obtenir le plus souvent des cas de dysgénésie presque totale, le $13^{\mathrm{e}}$ jour correspondant à des destructions dans l'ensemble incomplètes, mais cependant toujours graves.

La réactivité des ovaires aux gonadotrophines et l'activité hypophysaire des femelles dysgénésiques ont été étudiées chez des animaux issus de mères injectées le $15^{\mathrm{e}}$ jour de la gestation.

\section{Aptitude à la reproduction des femelles dysgénésiques.}

Les femelles dysgénésiques sont laissées en présence de mâles normaux (I mâle pour 2 femelles) pendant 3 mois.

Dans le cas de femelles issues de mères injectées le $13^{e}$ jour de la gestation, quatre femelles sur 30 se sont reproduites. Une d'entre elles a accouché normalement, les deux autres ont eu des difficultés et les petits n'ont pas survécu.

Dans le cas de femelles issues de mères injectées le $15^{e}$ jour aucune femelle n'a été gravide sur 30 . 
2. Etude des effecteurs.

a) Frottis vaginaux. - Ils ont été effectués pendant un mois, sur des rattes âgées de 80 jours environ et se sont toujours révélés atypiques.

Sur 20 femelles issues de mères injectées le $13^{e}$ jour de la gestation, $I 5$ ont présenté pendant un mois un frottis constitué de cellules kératinisées et de cellules épithéliales nucléées caractéristiques du pro-œstrus. Il arrive en outre que ces images soient interrompues pendant 2 ou 3 jours par un frottis de diostrus ou de vrai œestrus. Les 5 autres frottis étaient du type diœestrus constant.

Sur 20 femelles issues de mères injectées le $15^{\mathrm{e}}$ jour de la gestation, Io ont présenté un diœstrus permanent, 4 un diœstrus parfois interrompu par 2 jours d'œestrus, 6 enfin avaient un frottis de pro-œstrus intercalé de 5 jours d'œestrus.

b) Muqueuse vaginale. - Les muqueuses vaginales des femelles dysgénésiques témoignent d'une imprégnation hormonale dans la majorité des cas :

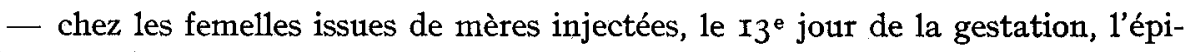
thélium est de type pavimenteux stratifié et souvent kératinisé ou parfois de type diœstrus. A 7 mois, chez tous les animaux sacrifiés, l'épithélium vaginal est pavimenteux stratifié ;

- les vagins prélevés sur des femelles issues de mères injectées le $15^{\text {e jour de }}$ la gestation, âgées de 2 mois sont dans la majorité des cas peaucistratifiés avec couche superficielle mucifiée, comme 1'a montré la coloration du Muci-carmin. Plus rarement, nous avons trouvé des images de kératinisation. A 7 mois, par contre, tous les animaux observés présentent une muqueuse vaginale de type pavimenteux stratifié parfois kératinisé.

c) La muqueuse utérine présente un épithélium très haut et souvent même stratifié. A 7 mois on note une hypertrophie glanđulo-kystique avec métaplasie pavimenteuse des glandes.

\section{Réactivité aux gonadotrophines.}

Lorsque des ovaires dysgénésiques de femelles issues de mères injectées le

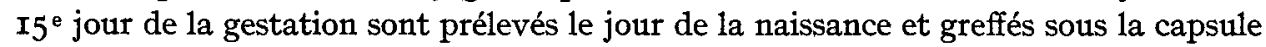
rénale de femelles adultes normales castrées le jour de la greffe, le greffon, I5 jours après l'opération est toujours de très petite taille et constitué essentiellement de structures cellulaires en cordons et en tubes, au sein d'un tissu théco-interstitiel stimulé.

Lorsque le greffon est resté en place I mois, on peut y observer des plages de cellules d'aspect lutéal. Dans un seul cas, nous avons trouvé de véritables corps jaunes.

Les muqueuses vaginales des femelles porte-greffes n'ont pas réagi. La production hormonale n'étant pas suffisante pour stimuler les effecteurs in situ, nous avons réalisé des greffes associées d'ovaires et d'effecteurs (vagin et vésicule séminale impubère) afin d'apprécier l'action hormonale de la gonade greffée et la quantité d'hormone sécrétée.

Au bout de I5 jours d'expérience, le fragment de vagin greffé au contact de l'ovaire présente parfois des signes de stimulation, mais toujours très faible. Nous n'avons jamais pu observer, des images nettes de kératinisation ou de mucification 
comme dans le cas de greffes témoins réalisées avec des ovaires normaux (BousQuETVANHEMS, I970).

Les greffes associées d'ovaires dysgénésiques et de vésicules séminales n'ont pas permis de détecter la présence d'androgènes en quantité suffisante.

\section{Activité hypophysaire.}

Lorsque des ovaires normaux prélevés le jour de la naissance sont greffés sous la capsule rénale de femelles dysgénésiques âgées de 70 jours et gardant leurs ovaires, le greffon I5 jours après l'opération est toujours très volumineux et bien conservé. A 1'examen microscopique, on constate qu'il est constitué de follicules à tous les stades de leur évolution au sein d'un parenchyme fortement stimulé. Les follicules primordiaux sont rares et toujours localisés en bordure du greffon. Le tissu thécointerstitiel, hyperplasique, forme de gros nodules s'insinuant entre les follicules, constitués essentiellement de grosses cellules d'aspect lutéal et organisées parfois en " corps jaunes" à ovules inclus.

L'examen histologique des effecteurs vaginaux, utérins et mammaires des rattes dysgénésiques porte-greffe, permet de constater l'existence d'une hyperstimulation œstrogénique. L'épithélium vaginal est pavimenteux stratifié, kératinisé (type œstrus), l'endomètre montre un état hyperœstrogénique marqué avec un épithélium formé de cellules très hautes, la glande mammaire présente un léger développement canaliculaire.

Lorsque le greffon est resté en place un mois, il est essentiellement constitué de volumineux corps jaunes. $L_{a}$ structure histologique des effecteurs du portegreffe est un peu différente de celle observée après I5 jours de greffe : l'épithélium de la muqueuse vaginale est pavimenteux stratifié, non kératinisé.

La comparaison de ces greffons avec ceux obtenus dans le cas de témoins permet de noter une différence considérable de l'évolution de l'ovaire transplanté. La greffon témoin est toujours beaucoup moins volumineux, le tissu théco-interstitiel ne se développe pas, même après un mois de greffe.

Chez les rattes porte-greffon les ovaires dysgénésiques en place sont dans tous les cas semblables à ceux déjà décrits. On n'y trouve jamais de follicules. Ils ne sont pratiquement constitués que de cordons cellulaires et pauvent présenter un ou deux petits corps jaunes.

\section{DISCUSSION ET CONCLUSIONS}

Les femelles issues de rattes traitées par le Misulban pendant la grossesse, présentent des atteintes ovariennes très graves si l'injection est réalisée entre le $12^{\mathrm{e}}$ et le $\mathrm{I} 6^{\mathrm{e}}$ jour de la gestation, les dysgénésies les plus importantes étant obtenues lorsque le produit est injecté le $15{ }^{e}$ jour.

Ces résultats concordent avec ceux de BEAUMONT (I96I) qui a montré que c'est aussi au $15^{\mathrm{e}}$ jour de la vie foetale que les cellules germinales de la Ratte sont le plus radio-sensibles, cette date d'après BEAUMONr et MANDL (r962) correspondant au moment de la dernière division mitotique des cellules germinales au sein de la gonade et expliquant la vulnérabilité maximale des gonocytes aux rayons et aux substances radiomimétiques. 
D'autre part, les aspects variables de l'atteinte ovarienne, non seulement en fonction du jour de l'injection de Misulban, mais aussi pour un même jour, d'un animal à l'autre et souvent même entre Rattes de la même portée peut s'expliquer par le fait que tous les gonocytes ne sont pas au même stade de développement au moment de l'injection, ce qui peut entraîner une différence de sensibilité au Misulban.

L'ovaire dysgénésique présente toujours une diminution de volume qui peut affecter divers degrés, sa structure histologique étant aussi modifiée. En l'absence d'ovocytes, l'organisation folliculaire ne se fait pas et l'ovaire conserve sa structure primaire. Il est constitué de formations cellulaires épithéliales organisées en travées à disposition radiaire en doigts de gant. Ces images correspondent à celles observées sur des ovaires de Rats ou de Souris après irradiation des fœetus (RAYNAUd, Ig66). L'allure de ces cordons rappelle aussi l'épithélium germinatif constituant l'ovaire d'Opossum à $\mathrm{I}$ mois (BuRNs, I956).

Quand toutes les cellules germinales n'ont pas été détruites, des follicules s'organisent. Certains subissent une maturation apparemment normale et peuvent évoluer jusqu'aux corps jaunes, mais beaucoup aussi involuent sur le mode atrétique.

Les nodules thécaux observés rappellent les résultats de Moraro-Manaro et ANBRIEUX (194I) qui, à la suite d'injection de gonadotrophines à des femelles de Cobaye infantiles ont obtenu une prolifération de la thèque. Ils ont précisé que ce tissu produisait des androgènes.

Le stock des follicules primordiaux qui, normalement représente le potentiel évolutif devant servir tout au long de la vie génitale est ici vite épuisé, et très tôt donc l'ovaire est d'apparence sénile. L'architecture cordonnale qu'il conserve avec la présence entre les cordons épithéliaux de volumineuses cellules qui semblent très actives, rappelle les images d'ovaire de Taupe observées en période anœestrale et décrites par DeANESLy (I935), ainsi que celles d'ovaires du Desman décrites par PEYRE (r952).

Les images de cordons cellulaires au sein desquels on reconnaît deux types différents de cellules, posent le problème de "l'évolution " de ces structures épithéliales en l'absence d'organisation folliculaire.

Ces ovaires dysgénésiques répondent aux stimulations pituitaires gonadotropes de l'hypophyse de rattes castrées sur lesquelles ils sont transplantés, en fonction des constituants qu'ils possèdent au moment de la greffe : si les ovaires ne contiennent que des cordons cellulaires, leur image n'est guère modifiée. S'ils contiennent de rares follicules, ceux-ci sont stimulés et peuvent se transformer en petits corps jaunes. Le fait que les femelles atteintes de dysgénésies gonadiques, provoquées par injection

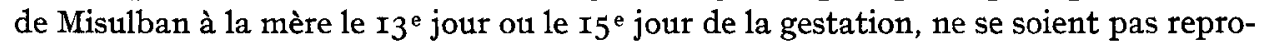
duites dans l'ensemble peut s'expliquer par le fait que le capital folliculaire était nettement insuffisant ou inexistant, les cas exceptionnels de gravidité correspondant à des atteintes moins graves de l'ovaire.

Les frottis vaginaux d'une part, la structure des effecteurs d'autre part, témoignent dans presque tous les cas d'une anomalie des cycles ; mais les conditions hormonales sont difficilement interprétables à cause des différentes images rencontrées.

Dans l'ensemble, on peut penser qu'il existe une déficience œestrogénique de l'ovaire accompagnée d'une hypersécrétion d'hormones gonadotropes qui se manifeste au niveau du greffon d'ovaire normal dans le rein de femelles dysgénésiques. 
Rappelons que des taux élevés d'hormones gonadotropes sont aussi retrouvés en clinique dans les cas de dysgénésies gonadiques et dans les ménopauses précoces (FRANCHIMONT, I97I).

On peut penser d'autre part que l'épithélium vaginal pavimenteux stratifié, la métaplasie pavimenteuse utérine retrouvée dans tous les cas à l'âge de 7 mois chez les animaux porteurs d'ovaires constitués d'amas de cellules très grosses et qui semblent très actives proviennent d'une stimulation continue de l'ovaire par LH qui en réponse fournirait des stéroïdes de façon constante et sans doute de type androgène (JAYis, Mauvais-JaRvis, I965).

\section{REMERCIEMENTS}

R. C. P. 221 du C. N. R.S.

Nous tenons à remercier les Laboratoires Techni - Pharma qui ont bien voulu mettre à. notre disposition la substance pure de Misulban.

\section{SUMMARY}

\section{EXPERIMENTAL STUDY OF RATS IN WHICH OVARIAN DYSGENESIA WAS PROVOKED DURING EMBRYONIC DEVELOPMENT}

Dysgenesia of the ovaries was produced by the destruction of the germinal cells during the embryonic stage by injecting the pregnant rat with Misulban.

In the absence of ovocytes, follicular organization does not take place and cordal structures are observed in the ovary which persist even after puberty.

In the event that germinal cells still exist, follicles evolve in which thecal hypertrophy is usually observed. The theca-interstitial tissue increases with the age of the animal, whereas the cordal structures diminish or disappear. Vaginal smears, as well as the structure of the targetorgans, display an abnormal hormonal impregnation. These dysgenesic ovaries, grafted into the kidney of castrated female rats, respond to gonadotrophic stimulation according to their constituents at the time of the graft. The pituitary functioning of these animals, tested by the grafting method, shows gonadotrophic hypersecretion.

\section{RÉFÉRENCES BIBLIOGRAPHIQUES}

Beaumont H. M., I96r. The radiosensitivity of oogonia and oocytes in the foetal Rat. Int. J. Radiation Biol., 3, 59-72.

Beaumont H. M., Mandi A. M., I962. A quantitative and cytological study of oogonia and oocytes in the foetal and neonatal Rat. Proc. Roy. Soc. B., 155, 557-559.

Bousquet J., Vanhems E., I970. Réactions des ovaires de Rat prélevés en période périnatale et greffés sur adultes. Évolution après passage en culture. C. R. Acad. Sci. Paris, 271, I638-1641.

Burns R. K., Burns L. M., I956. Vie et reproduction de l'Opossum américain, Didelphis marsupialis virginiana KERR. Bull. Soc. Zool. France, 81, 230-246.

DEANESLY R., I935. The reproductive processus of certain mammals. Part IX. Growth and reproduction in the Stoat (Mustila erminea). Phil. Trans. Royal Soc. London, séries B, 225, 459-492.

Diamond I., Anderson M., Creadie Mc. S., I96o. Transplacental transmission of busulfan (Myleran) in a mother with leukemia. In : Production of foetal malformations and cytomegaly. Pediatrics, 25, $85-90$.

Forsberg J. G., Olivecrona H., r966. The effect of prenatally administered Busulfan on Rat gonads. Biologia Neonat. 10, I80. 
Franchimont P., rg7r. Sécrétion normale et pathologique de la somatotrophine et des gonadotrophines humaines. I vol., 306 pages, Masson et Cle, Paris.

Gerlinger P., r966. Contribution à l'étude de l'action tératogène du cyclophosphamide chez l'embryon de Poulet et de Lapin. Arch. Anat. Histol. Embryol. norm. exp., 49, 249-3I7.

Heller R. R., Jones H. W., I963-r964. Experimental production of ovarian dysgenesis by Busulfan in. Anmual report depart. embryol. Carnegie Instit., 564.

Hemsworth B. N., Jackson H., r962. Effect of Busulfan on the foetal gonad. Nature, Lond., $185,8 \mathrm{r} 6$.

Hemsworth B. N., Jackson H., I963. Effect of Busulfan on the developing ovary in the Rat. $J$. Reprod. Fert., 6, 229.

Hemsworth B. N., Jackson H., 1965. Embryopathies induced by cytotoxic substances. In : $A$ Symposium on Embryopathic Activity of Drugs. Edited by J. M. Robson, F. Sullivan and R. L. Smith, J. A. Churchill Ltd, London.

HeMsworth B. N., r969. Effect of alkane sulphonic esters on ovarian development and function Rat. J. Reprod. Fert., 18, 15-20.

Jayle M. F., Mauvais-Jarvis P., 1965. Anomalies de la biogenèse et du métabolisme des hormones stéroïdes dans les affections congénitales ovariennes. Ann. Endoc., Paris, 25, 750-752.

MANDL A. M., Shelton M., I959. A quantitative study of oocytes in young and old multiparous laboratory Rats. J. Endocr., 18, 444-450.

MANDL M., I963. The radiosensitivity of oocytes at different stages of maturation. Proc. Roy. Soc. (B), 158, II9-I4I.

MANDL M., I964. The radiosensitivity of germ cells. Biol. Rev., 38, 288-371.

Mauss H. J., MeYer K., r964. Vergleichende Untersuchungen über dir Wirkung des zytostatischen Trenimon und Myleran auf foetal ratten ovarian. Zentralblatt $f$. Gynäk., 86, 72-78.

Mintz B., 1958. Irradiation of primordial germ cells in the Mouse embryo. Anat. Rec., 130, $34 \mathrm{I}$.

Morato-Manaro J., Albrieux A., i94I. Masculinisation de Cobayes femelles infantiles par le Prolan. Ann. Endocr., 2, 93.

Pageot-Simpson P., WolfF E., 1969. La sensibilité différentielle d'une tumeur maligne humaine et des gonades embryonnaires en culture organotypique in vitro à une substance alkylante le Melphalan. C. R. Acad. Sci., 268, 2997-3000.

Peyre A., r952. Note sur la structure histologique de l'ovaire du Desman des Pyrénées, Galemys Pyrenaicus G. Bull. Soc. Zool. France, 77, 44I-446.

Pinto-Machado, I967. The influence of busulfan ( $\mathrm{x}-4$ dimethanesulfonyloxybutane) on the prenatal development of the gonad of Mice. C. R. Ass. Anat., 139, 1024-1030.

Raynaud A., I966. Effet des rayons $\mathrm{X}$ sur l'embryon et le foetus des mammifères. Radiobiologie appliquée. Gauthier-Villars éd., Paris, 513-58I.

Raynaud A., Frilley M., r945. Irradiation au moyen des rayons $\mathrm{X}$ des ébauches des glandes génitales de l'embryon de Souris au $5_{5}{ }^{\mathrm{e}}$ jour de la vie intrautérine. C. R. Acad. Sci. Paris, 223, I-137.

Raynaud A., Frilley M., 1947. Destruction des glandes génitales de l'embryon de Souris par une irradiation au moyen des rayons $\mathrm{X}$ à l'âge de $\mathrm{I} 3$ jours. Ann. endocr., 8, 400 .

Rugh R., I952. Foetal X irradiation and fertility. Proc. Soc. Exp. Biol. Med., 80, 388-395.

Rugh R., Jackson S., 1958. Effect of foetal $X$ irradiation upon the subsequent fertility of the offspring. J. Exp. Zool., 138, 209-221. 


\section{PLANCHE I}

FIG. I

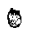

Ovaire dysgénésique de Io jours. On observe un follicule primordial au sein de l'ovaire constitué par ailleurs de cordons cellulaires qui en l'absence d'autres ovocytes ne se sont pas fragmentés. $\mathrm{G}: \times 25$

FIG. 2

Hypertrophie thécale dans un ovaire de 20 jours. $G: \times 40$

8

\section{FIG. 3}

Ovaire dysgénésique de I mois.

Détail des cordons cellulaires qui n'ont pas évolué. $G: \times 25$

\section{FIG. 4}

Ovaire de 50 jours. Présence de nodules thécaux donnant à l'ơaire un aspect très dense. $G: \times 4$

FIG. 5

Détail de la figure $4 . \mathrm{G}: \times 25$

FIG. 6

Ovaire dysgénésique de 3 mois. Développement du tissu thécointerstitiel entre les cordons cellulaires. $\mathrm{G}: \times$ Io 

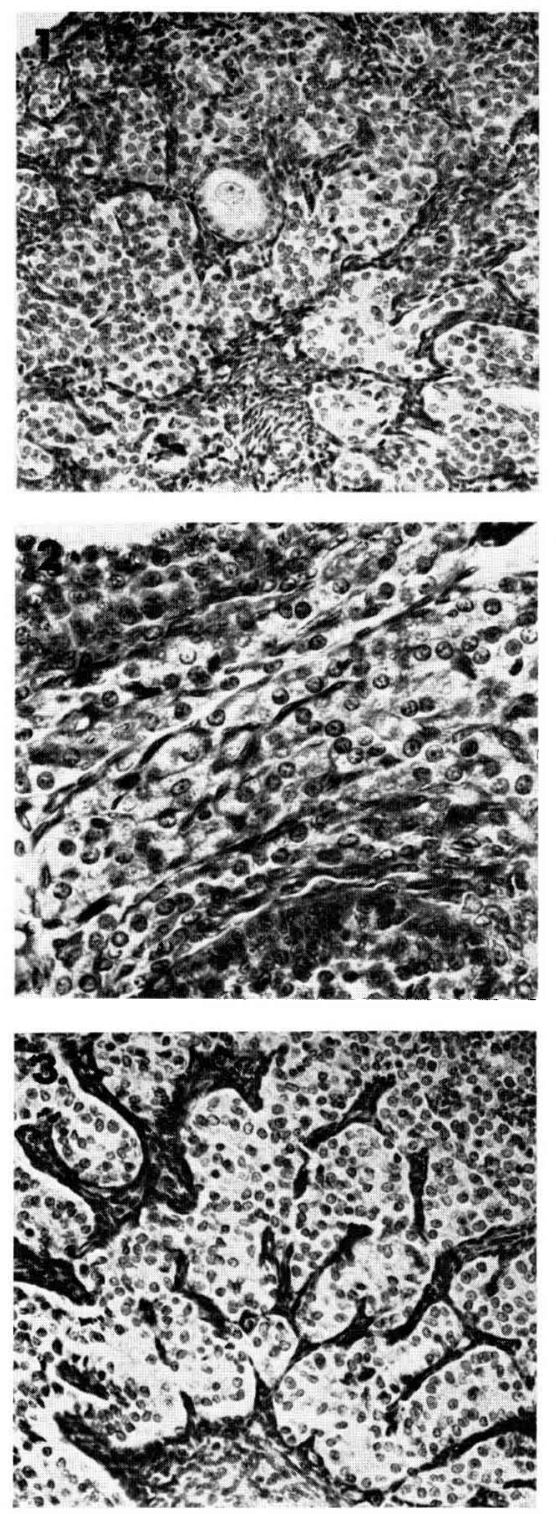
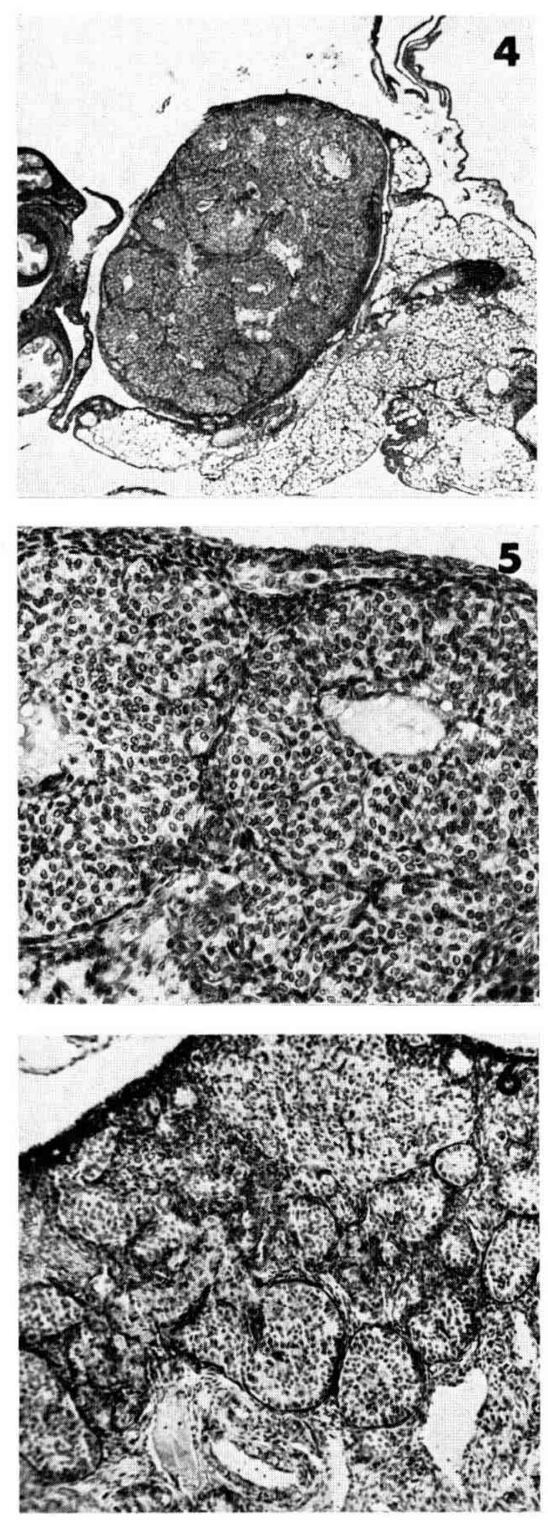


\title{
PLANGHE II
}

\author{
FIG. 1
}

Détail d'un cordon cellulaire au sein d'un ovaire dysgénésique de 3 mois. Présence de volumineuses cellules. G : $\times 100$.

FIG. 2

Parenchyme ovarien de 7 mois, constitué de deux types cellulaires, les uns à limite cytoplasmique nette, à cytoplasme pâle, granuleux, et à noyau excentré, les autres à cytoplasme dense et bien coloré. G : $\times 100$.

\section{Fig. 3}

Coupe d'une corne utérine d'une ratte âgée de 7 mois. Hyperplasie glandulo-kystique typique, métaplasie pavimenteuse des glandes utérines. $\mathrm{G}: \times 10$.

FIg. 4

Greffe intrarénale d'ovaire dysgénésique du jour de la naissance chez une femelle normale castrée le jour de la greffe. Après 15 jours de greffe les structures cordonnales n'ont pas évolué. $\mathrm{G}: \times 40$.

FIG. 5

Greffe intrarénale d'ovaire normal du jour de la naissance chez une femelle dysgénésique, 15 jours après, le greffon est très volumineux. Le tissu interstitiel hyperplasique organisé en corps jaunes à ovule inclus témoigne d'une hyperstimulation gonarotrope. $\mathrm{G}: \times 4$.

FIG. 6

Détail de la figure 5 . Corps jaune à ovule inclus. G : $\times 40$. 
PLANCHE II
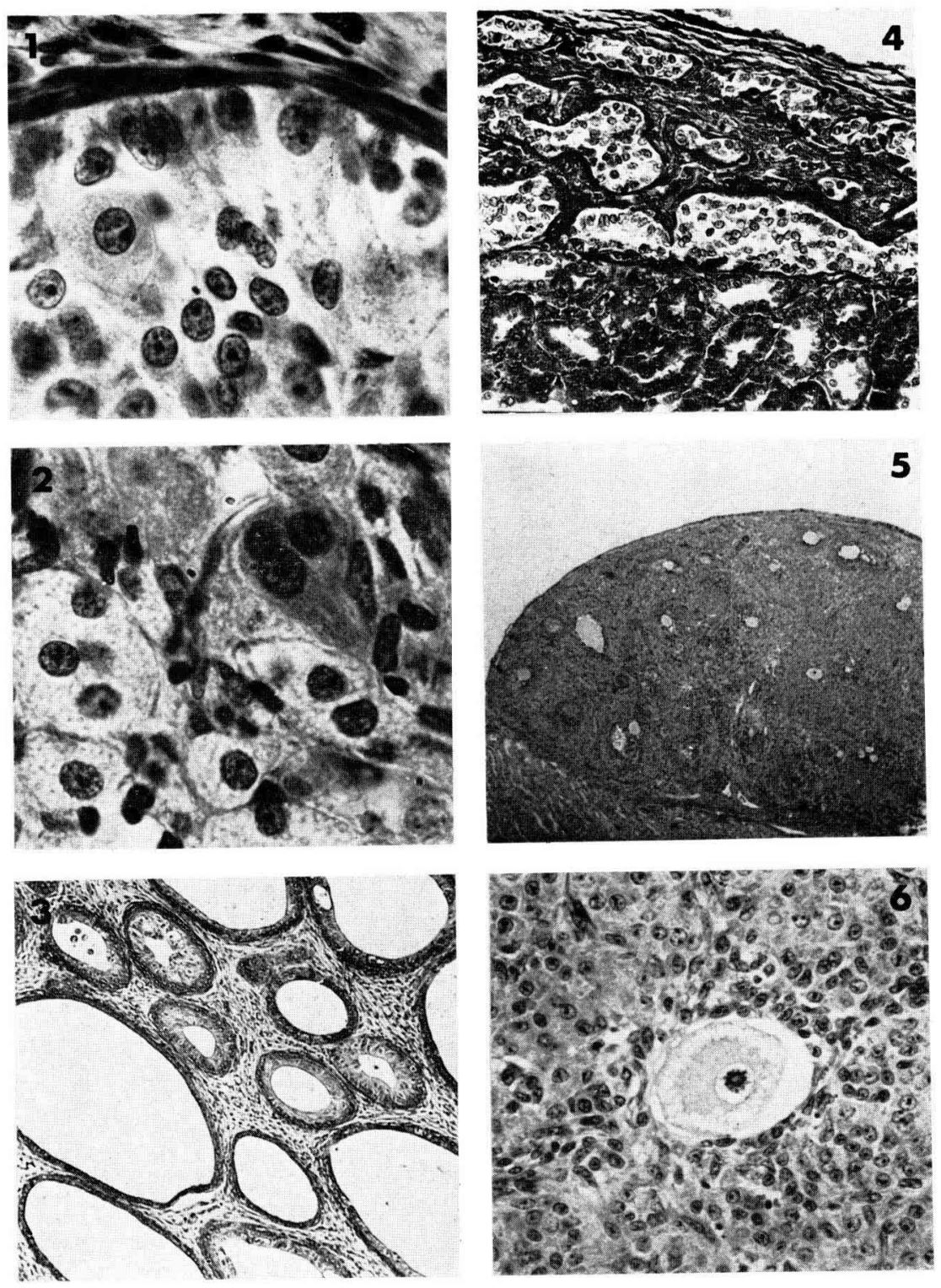